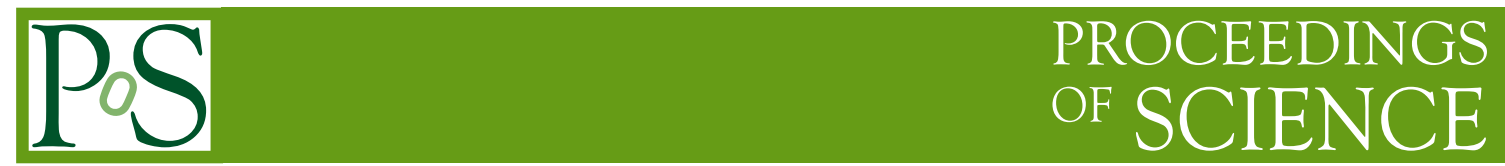

\title{
New particles at Belle
}

\author{
Pavel Pakhlov*i \\ ITEP, Russia \\ E-mail: pakhloveitep.ru
}

\begin{abstract}
We report the observation of new decay modes of the $X(3872)$ and the study of possible $J^{P C}$ quantum number assignments for this state. We present observations of new charmonium states, produced in different processes: $B$ decays, $e^{+} e^{-}$annihilation and $\gamma \gamma$ interactions. These results are obtained from a large data sample collected with the Belle detector near the $\Upsilon(4 S)$ resonance, at the KEKB asymmetric-energy $e^{+} e^{-}$collider.
\end{abstract}

International Europhysics Conference on High Energy Physics

July 21st - 27th 2005

Lisboa, Portugal

${ }^{*}$ Speaker.

${ }^{\dagger}$ on behalf of the Belle Collaboration. 


\section{Introduction}

Recently there have been a number of reports of new charmonium or charmonium-like states at Belle: $\eta_{c}(2 S), X(3872), Y(3940)$ [1]. The first state, $\eta_{c}(2 S)$, was identified reliably both by its discovery decay mode and by its production in $\gamma \gamma$ collisions. The $X(3872)$ decay into $J / \psi \pi \pi$ suggests it can be treated as a typical charmonium particle. However, its observed mass and narrow width makes the assignment to any of charmonium states problematic. Moreover, the mass of the dipion system tends to concentrate near the mass of the $\rho(770)$ meson, while the decay of a charmonium state to $J / \psi \rho$ would violate isospin and should be strongly suppressed. Finally, another state, $Y(3940)$, was observed in $B$ decays in the $J / \psi \omega$ final state and again has no conventional explanation. In this report we describe recent results on the $X(3872)$, and discuss the observations of new charmonium states in the Belle experiment.

\section{New $X(3872)$ decay modes and constrain on $J^{P C}$}

If the dipion system in the $X(3872) \rightarrow J / \psi \pi \pi$ decays indeed forms a $\rho(770)$, other isospin violating modes such as $X(3872) \rightarrow J / \psi \gamma$ and $X(3872) \rightarrow J / \psi \pi^{+} \pi^{-} \pi^{0}$ can be large enough for experimental detection. In our search for the first mode, we study $B \rightarrow J / \psi \gamma K$ signal yields in bins of the $M(J / \psi \gamma)$ and find an enhancement around the $X(3872)$ mass (Figure. 1 a). The statistical significance of the observation of the $X(3872) \rightarrow J / \psi \gamma$ decay is estimated to be $4.0 \sigma$. We calculate the ratio of the partial widths to be:

$$
\mathscr{B}(X(3872) \rightarrow J / \psi \gamma) / \mathscr{B}(X(3872) \rightarrow J / \psi \pi \pi)=0.14 \pm 0.05
$$

We also observe strong evidence for the decay $X(3872) \rightarrow J / \psi \pi^{+} \pi^{-} \pi^{0}$. For the $J / \psi \pi^{+} \pi^{-} \pi^{0}$ combinations selected around $X(3872)$ mass, we study $B \rightarrow J / \psi \pi^{+} \pi^{-} \pi^{0} K$ signal yields in bins of mass of $\pi^{+} \pi^{-} \pi^{0}$ system. A significant signal is observed for a single bin around the kinematical limit between $750 \mathrm{MeV}$ and $775 \mathrm{MeV}$ (Figure. $1 \mathrm{~b}$ ), suggesting that the process is dominated by
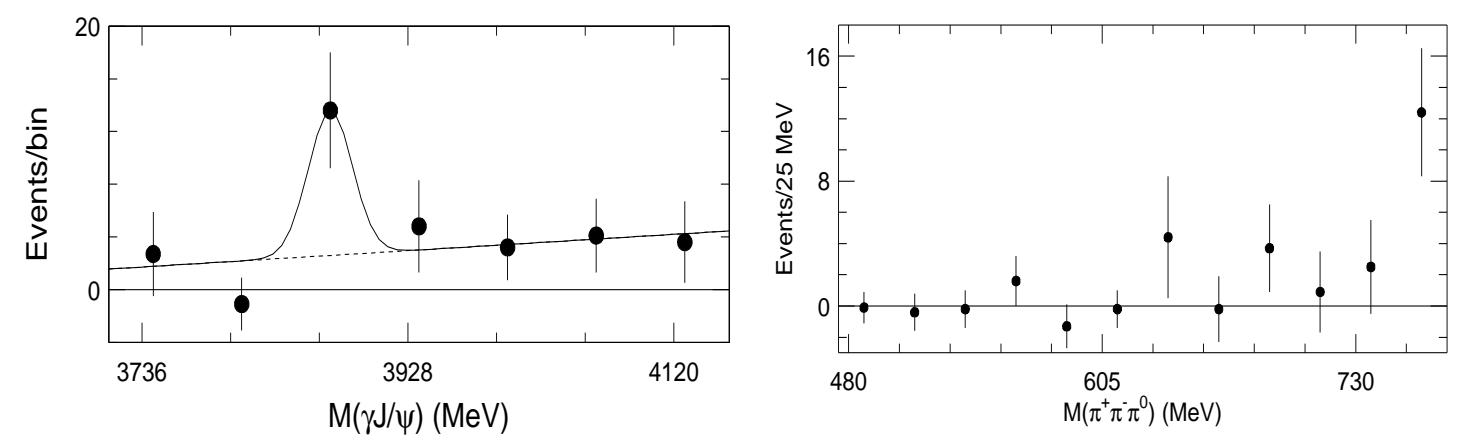

Figure 1: a) The $B$ yields in bins of $M(J / \psi \gamma)$; the solid line represents the fi t. b) The $B$ yields in bins of $M\left(\pi^{+} \pi^{-} \pi^{0}\right)$.

the sub-threshold decay $X(3872) \rightarrow J / \psi \omega$. These results establish the charge-conjugation parity of the $X(3872)$ as $C=+1$ [2].

We examine possible $J^{P C}$ quantum number assignments for the $X(3872)$ [3]. Angular correlations between final state particles in $X(3872) \rightarrow J / \psi \pi^{+} \pi^{-}$decays are used to eliminate $J^{P C}$ 
values of $0^{++}$and $0^{-+}$. The shape of the $\pi^{+} \pi^{-}$mass distribution near its upper kinematic limit favors $S$-wave over $P$-wave as the relative orbital angular momentum between the dipion and $J / \psi$, which strongly disfavors $1^{-+}$and $2^{-+}$. The accumulated evidence strongly favors a $J^{P C}=1^{++}$ assignment for the $X(3872)$, although the $2^{++}$possibility is not ruled out by the tests reported here.

\section{Observation of a new charmonium-like state produced in association with $J / \psi$ in $e^{+} e^{-}$annihilation}

We report the first observation of a new charmonium-like state at a mass of $\sim 3.94 \mathrm{GeV} / c^{2}$. This state, which we denote as $X(3940)$, is observed in the spectrum of masses recoiling against the $J / \psi$ in the inclusive process $e^{+} e^{-} \rightarrow J / \psi+$ anything. The recoil mass spectrum for the inclusive $J / \psi$ event sample is shown in Figure 2 a. Here, in addition to the three previously reported peaks at the $\eta_{c}, \chi_{c 0}$ and $\eta_{c}(2 S)$ masses [4], there is a fourth peak above the $D \bar{D}$ threshold. The significance of the latter peak is estimated to be $5.0 \sigma$.

After reconstruction of a $D$ meson in the inclusive $J / \psi$ events and constraint of recoil mass against the $J / \psi D$ system into the nominal $D^{*}$ mass, a peak with better resolution over a smaller background can be seen in the spectrum of masses recoiling against the $J / \psi$ (Figure $2 \mathrm{~b}$ ). The sig-
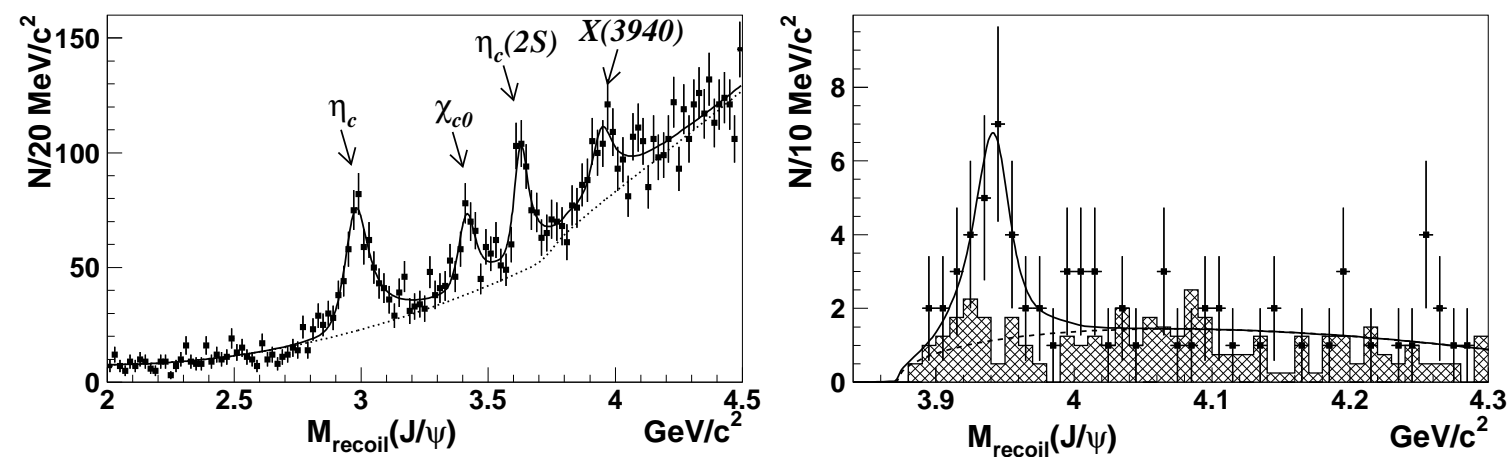

Figure 2: a) The $M_{\text {recoil }}(J / \psi)$ distribution in inclusive $e^{+} e^{-} \rightarrow J / \psi X$ events. b) The $M_{\text {recoil }}(J / \psi)$ distribution for events tagged and constrained as $e^{+} e^{-} \rightarrow J / \psi D^{*} \bar{D}$. The hatched histogram shows scaled $D$ sidebands. The solid line is the result of the fi $t$; the dashed line is the background function.

nifcance of the signal is $5.0 \sigma$. The extracted $X(3940)$ parameters are $M=(3943 \pm 6 \pm 6) \mathrm{MeV} / c^{2}$ and $\Gamma<52 \mathrm{MeV} / c^{2}$ at the $90 \%$ C.L.. We calculate the fraction of $X(3940)$ decays into $D^{*} \bar{D}$ among all found in the inclusive $J / \psi$ recoil mass spectrum to be:

$$
\mathscr{F}\left(X(3940) \rightarrow D^{*} \bar{D}\right)=\left(96_{-32}^{+45} \pm 22\right) \%,
$$

suggesting the decay $X(3940) \rightarrow D^{*} \bar{D}$ to be the dominant mode. In addition, no signal of $X(3940) \rightarrow$ $D \bar{D}$ and $X(3940) \rightarrow J / \psi \omega$ is found [5].

\section{Observation of a new charmonium state in $\gamma \gamma$ interactions}

We report on a search for the production of new resonance states in the process $\gamma \gamma \rightarrow D \bar{D}[6]$. The studied process is selected using a set of requirements based on the $P_{t}$ of the final state candidate, visible energy and mass in the event. The invariant mass of the combined $D^{0} \bar{D}^{0}$ and $D^{+} D^{-}$ 
channels is shown in Figure $3 \mathrm{a}$. A peak at a mass of $\sim 3.93 \mathrm{GeV} / c^{2}$ is clearly visible and we denote this state as $Z(3930)$. The significance of the peak is estimated to be $5.5 \sigma$. The $Z(3930)$ parameters extracted from the fit are $M=(3931 \pm 4 \pm 6) \mathrm{MeV} / c^{2}$ and $\Gamma=(20 \pm 8) \mathrm{MeV} / c^{2}$.

To identify the state we study the $Z(3930)$ decay angular distribution. Figure $3 \mathrm{~b}$ shows the
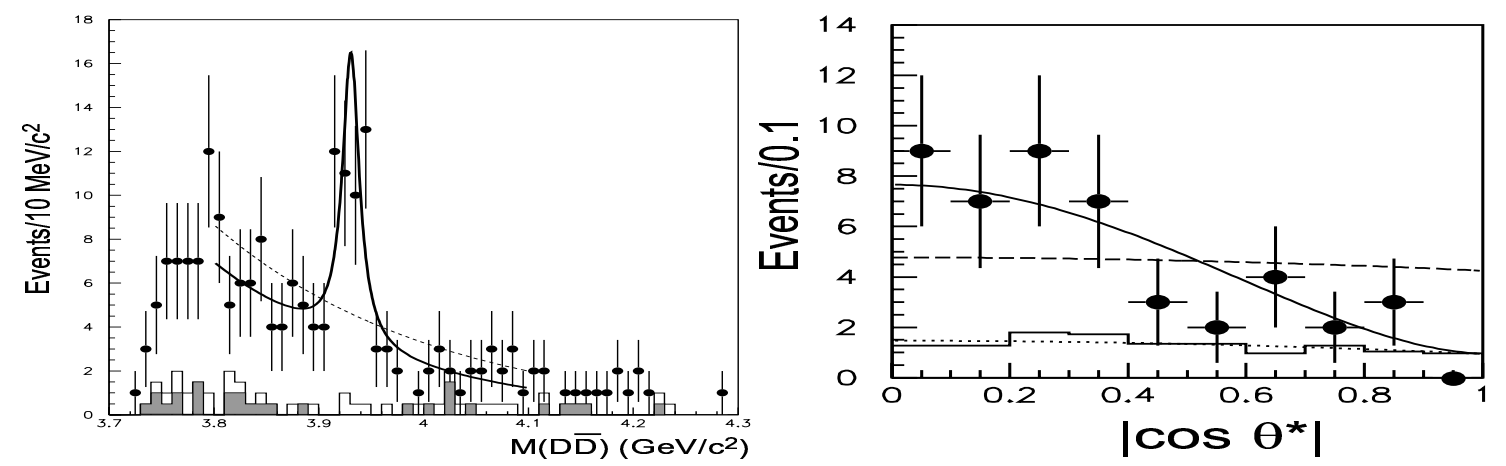

Figure 3: a) The $M(D \bar{D})$ distribution (points with error bars); the histograms correspond to scaled $D$ sidebands. The solid and dashed lines are results of fi $\mathrm{t}$ with and without resonance component. $\mathrm{b})$ The $\cos \left(\theta^{*}\right)$ distribution for events from the $Z$ (3930) signal region (points with error bars) and $Z(3930)$ sidebands (histogram). The solid and dashed lines are the expected distributions for spin-2 and spin-0 particles.

$\cos \theta^{*}$ distribution for the events selected from the $Z(3930)$ signal region, where $\theta^{*}$ is the angle of a $D$ meson relative to the beam axis in the $\gamma \gamma$ center-of-mass frame. For a spin- 0 resonance this distribution should be flat, while for a spin-2 resonance it is expected to be $\sim \sin ^{4} \theta^{*}$. Thus our data favors that this state is the previously unobserved $\chi_{c 2}^{\prime}$, the $2^{3} P_{2}$ charmonium state. Finally, we calculate the $\Gamma_{\gamma \gamma}$ width of the state, assuming $J=2$, to be:

$$
\Gamma_{\gamma \gamma} \times \mathscr{B}(Z(3930) \rightarrow D \bar{D})=(0.23 \pm 0.06 \pm 0.04) \mathrm{keV}
$$

\section{Summary}

We present the observation of the $X(3872)$ decay into $J / \psi \gamma$ and $J / \psi \pi^{+} \pi^{-} \pi^{0}$ and the systematic study of the $X(3872)$ properties. The new charmonium-like states are observed for the first time in $e^{+} e^{-}$anihillation and $\gamma \gamma$ collision.

\section{References}

[1] S.K. Choi et al. (Belle Collaboration), Phys. Rev. Lett. 89, 102001 (2002);

S.K. Choi et al. (Belle Collaboration), Phys. Rev. Lett. 91, 262001 (2003);

S.K. Choi et al. (Belle Collaboration), Phys. Rev. Lett. 94, 182002 (2005).

[2] K. Abe et al. (Belle Collaboration), hep-ex/0505037.

[3] K. Abe et al. (Belle Collaboration), hep-ex/0505038.

[4] K. Abe et al. (Belle Collaboration), Phys. Rev. Lett. 89, 142001 (2002).

[5] K. Abe et al. (Belle Collaboration), hep-ex/0507019, submitted to Phys. Rev. Lett.

[6] K. Abe et al. (Belle Collaboration), hep-ex/0507033. 